\title{
Lista de corales hermatípicos del Museo Javeriano de Historia Natural Lorenzo Uribe S.J., Colombia
}

\author{
Martha Y. Vallejo' y Alberto Acosta PhD² \\ ${ }^{1,2}$ Pontificia Universidad Javeriana, Facultad de Ciencias, Departamento de Biología. Unidad de Ecología y Sistemática (UNESIS).

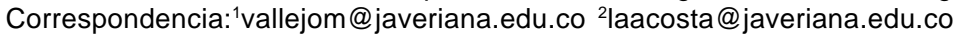 \\ Recibido: 27-04-2005 - Aceptado: 05-10-2005
}

Las colecciones biológicas de diferentes museos en un país son material complementario que sirven para estudios e investigaciones sobre biodiversidad, ya que además de la información sobre los especimenes preservados, ofrecen instrumentos para crear vínculos y relaciones entre sí. La presente nota, ilustra la importancia de la complementariedad entre colecciones: Museo Javeriano; MUJ y Museo de Historia Natural Marino de Colombia; MHNMC, para un mismo grupo biológico, los corales hermatípicos. La nomenclatura de especies utilizadas fue siguiendo a Veron (2000). El MUJ busca participar con su colección de referencia (29 especies, 129 registros biológicos) en proyectos nacionales e internacionales, compartiendo información, para de esta manera aportar al conocimiento y estudio de la biodiversidad.

Palabras claves: colección biológica, corales hermatípicos, museos, lista de especies.

Cada colección de un grupo biológico particular es única para el país o el mundo, ya que brinda información taxonómica y geográfica de sus ejemplares en escalas diferenciales de espacio y tiempo. Por lo tanto, las especies exclusivas de cada colección generan complementariedad taxonómica con otras colecciones y las especies que comparten se constituyen en material de respaldo para el país; esto en la eventualidad que parte o la totalidad de una colección se pierda.

La lista de una colección permite identificar presencia - ausencia de especies respecto a un marco de referencia (listado reportado para el país), así como para incrementar potencialmente el marco existente; maximizar la revisión taxonómica y bibliográfica de un grupo biológico, y como material de consulta y comparación (1).

El comparar las listas de especies de un grupo biológico de diferentes colecciones nacionales e interna- cionales, permite definir: la representatividad total de especies que reposan en los museos; su aporte a la biodiversidad, las unidades geográficas muestreadas, el estado de las colecciones y sus prioridades mediante la aplicación de índices de biodiversidad (1); así como gestionar el préstamo e intercambio de ejemplares entre colecciones. Pese a su importancia, los listados taxonómicos hasta el momento han contado con pocos espacios para su publicación y difusión, hecho que ha afectado considerablemente su aprovechamiento como herramienta de investigación e insumo para la toma de decisiones (1).

El listado de corales Escleractínios; Cnidaria: Anthozoa reportado para Colombia (2), incluye ejemplares que reposan en las colecciones de referencia del Museo de Historia Natural Marina de Colombia (MHNMC; Santa Marta - Colombia) y del National Museum of Natural History (NMNH; Smithsonian 
Institution, Washington D.C. - USA). Sin embargo, recientemente fueron referenciados para el Museo Javeriano de Historia Natural Lorenzo Uribe S.J., (MUJ, Bogotá - Colombia) los corales hermatípicos colectados en el Caribe Colombiano.

Esta nota reporta 129 registros biológicos y proporciona la lista de 29 especies de corales hermatípicos (tabla 1); determinados a partir de claves sinópticas siguiendo a Veron 2000 (3); y una guía visual de campo, usando caracteres diagnósticos para la identificación de corales juveniles $<4 \mathrm{~cm}$ de diámetro, datos no publicados, y únicos para el Caribe colombiano.

Adicionalmente se resalta la importancia de la información contenida en la lista de corales del MUJ por poseer: (i) Siete especies exclusivas y referenciadas para el país en la colección del Museo Javeriano; lo cual, por ser especies listadas por primera vez, genera alta complementariedad taxonómica, y alimenta la información existente entre las colecciones del MHNMC y el NMNH (4). (ii). Veintitrés especies de corales hermatípicos juveniles, 79\% de los ejemplares de la colección, que hacen de ésta exclusiva y única en el país.

De esta forma, los esqueletos y caracteres morfológicos diagnósticos del material referenciado facilitara la identificación de juveniles in situ en estudios futuros. La información contenida en la lista de corales del MUJ complementara otras colecciones marinas que manejan el mismo grupo biológico, lo cual dará una dimensión mas acertada de la biodiversidad de Colombia y facilitara futuras investigaciones.

Es importante precisar que en el listado de referencia de Reyes 2000 (2) incluye a Eusmilia fastigiata dentro de la familia Caryophylliidae aún cuando esta pertenece a Meandrinidae (3). De igual forma el autor sobreestima la biodiversidad de Colombia al reportar Leptoseris y Helioseris como géneros diferentes, cuando Veron (2000) y otros autores, como Cairns (1982) solo validan a Leptoseris (5).

Así mismo, para el Caribe, Veron (3) tan solo reporta a Leptoseris cucullata, quien ha sido denomi- nada en las colecciones como Helioseris cucullata en Colombia (6). Es importante señalar que el tener una lista de referencia para un grupo biológico no es suficiente si este no incluye el material presente en las diferentes colecciones del país.

\section{Agradecimientos}

Al profesor Camilo Andrade y sus alumnos del curso de invertebrados por la colaboración en la identificación de Scleractinios (MUJ). Al grupo de ecología de arrecifes de la PUJ (II-2003) por la identificación de corales juveniles hermatípicos. Este trabajo fue realizado dentro del proyecto "Organización y sistematización de las colecciones algales y de invertebrados no artrópodos de las colecciones biológicas del Museo Javeriano“ financiado por la Vicerrectoría de la Pontificia Universidad Javeriana (PUJ) Código $1583 / 2004$.

\section{Museo de Historia Natural Lorenzo Uribe S.J. Hermatypic Coral List, Colombia}

Each Biological collection in a particular group is unique, since it has taxonomic and geographic information of its specimens in different spatial and temporal scale. Therefore, exclusive species at each collection generate taxonomic complementarity with other collections, and shared species represent country's back up material in case of partial or total collection damage. Any collection species list allow us to: determine species presence-absence with respect to a referenced framework, reported list for the country, potential increment of the existing framework; maximize taxonomic and bibliographic revision of a biological group; and to search and compare specimens. Species list comparison of a biological group from different National and International collections permit us to define: total museum species representativity; biodiversity contribution, geographical sampled areas, collection's quality, collection's priorities based on biodiversity indexes (1); and to borrow and interchange specimens between 
Tabla 1. Corales esclaractinios del Caribe colombiano, referenciados en la colección del MUJ

\section{Taxón}

Acroporidae

Acropora cerviconis Lamarck, 1816

Acropora palmata Lamarck, 1816

Agariciidae

Agaricia agaricites Linnaeus, $1758 \Theta$

Agaricia tenuifolia Dana, $1846^{*} \Theta$

Agaricia sp

Leptoseris cucullata Ellis \& Solander, $1786 \Theta$

Astrocoeniidae

Stephanochoenia intersepta Esper 1795 \#Ө

tay cen dar san

Caryophylliidae

Cladocora arbuscula Leseur, 1881

Faviidae

Colpophyllia natans Houttuyn, $1772 \Theta$

Diploria clivosa Ellis \& Solander, 1786

Diploria labyrinthiformis Linnaeus, $1758 \Theta$

Diploria strigosa Dana, $1848 \Theta$

Favia fragum Esper, $1797 \Theta$

Manicina aereolata Linnaeus, $1758 \Theta$

Montastrea annularis Ellis \& Solander, $1786 \Theta$

Montastrea cavernosa Linnaeus, $1767 \Theta$

Montastrea faveolata Ellis \& Solander, 1786 * $\Theta$

Montastrea franksi Grogory, 1895*

Meandrinidae

Dichocoenia stokesi Milne, Edwars\& Haime, $1848 \Theta$

Meandrina meandrites Linnaeus, $1758^{\star} \Theta$

Eusmilia fastigiata Pallas, $1766{ }^{*} \Theta$

Mussidae

Isophyllastrea rigida Dana, $1846 \Theta$ tay cen san

gua tay mag cen dar san

tay mag cen dar san

gua tay cen dar san

tay

gua tay cen san

gua tay mag cen cas san

gua tay mag cen cas san

tay mag cen cas san

gua tay mag cen cas san

gua tay cen cas san

tay cen cas san

gua tay mag cen cas san

gua tay cen cas san

tay cen cas san

tay cen cas san

tay cen san

tay cen cas sen

tay cen cas san

cen cas san
MPUJ INV- 00102

MPUJ INV-00107-014:

MPUJ INV- 00038,000

MPUJ INV- 00129,001

MPUJ INV- 065

MPUJ INV- 0042,0004

MPUJ INV- 00106,001

MPUJ INV- 00008,001

MPUJ INV- 00054

MPUJ INV- 00154-001

MPUJ INV- 00150

MPUJ INV- 00091- 000

MPUJ INV- 00001,000

MPUJ INV- 00061-00

MPUJ INV- 00060,00

MPUJ INV- 00052-000

MPUJ INV- 00082-000

MPUJ INV- 00073-000

MPUJ INV- 00039,000

MPUJ INV- 00048,000

MPUJ INV- 00050,001

MPUJ INV- 00178

* Especies referenciadas exclusivas del MUJ. $\infty$ Sin datos disponibles. O Corales juveniles. \# Cairns et al. (1999) no la considera especie válida. / Abreviaturas. Museo Pontificia Universidad Javeriana. MPUJ-INV: Colección de referencia Museo Javeriano. Sección 


\section{3}

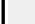

$140,0165,00074,00066$

77

$3,00047,00049,00064$

71

56,00159

$55,00015,00118,00127,00164$

092

$103,00045,00071,00075,00080,00122,00125,00126$

$162,00109,00157,00158,00170,00173-00174,00176$

$1072,00084,00085$

153

183

177

146,00144

151,00067

79
[7]Geyer 1969;[8] Pfaff 1969; [9]Antonius 1972; [10]Werding \& Sánchez 1989;[11] Sánchez 1995;[3]Veron 2000

Geyer 1969; Pfaff 1969; Antonius 1972; Werding \& Sánchez 1989;

[12]Ramirez et al. 1994; Veron 2000

Pfaff 1969; Antonius 1972; Werding \& Sánchez 1989; [13]Zea 1993; Ramirez et al. 1994 ; Veron 2000

[14]Erhardt 1974; Ramirez et al.1994;Sánchez 1995;Veron 2000

\section{Veron 2000}

Erhardt 1974; Zea 1993;Veron 2000

Pfaff 1969; Erhardt 1974; [15]Prahl \& Erhardt 1985; Ramirez et al. 1994 ; Veron 2000

Antonius 1972; Erhardt 1974; Werding \& Sánchez 1989; Ramirez et al. 1994; Veron 2000

Antonius 1972; Erhardt 1974; Werding \& Sánchez 1989; Ramirez et al.1994; Veron 2000

Płaff 1969; Antonius 1972; Erhardt 1974; Werding \& Sánchez 1989;

Sánchez 1995; Veron 2000

Pfaff 1969; Antonius 1972; Erhardt 1974; Zea 1993; Sánchez 1995; Veron 2000

Geyer 1969; Pfaff 1969; Antonius 1972; Erhardt 1974; Werding \& Sánchez 1989; Zea 1993; Ramirez et al.1994; Sánchez 1995; Veron 2000

Geyer 1969; Pfaff 1969; Antonius 1972; Ramirez et al. 1994;Veron 2000

Sánchez 1995; Veron 2000

Pfaff 1969; Erhardt 1974; Werding \& Sánchez 1989; Zea 1993;

Ramirez et al.1994; Sánchez 1995; Veron 2000

Sánchez 1995; Veron 2000

Sánchez 1995; Veron 2000

Pfaff 1969; Antonius 1972; Erhardt 1974; Werding \& Sánchez 1989; Ramirez et al.1994;Veron 2000

Geyer 1969; Pfaff 1969; Antonius 1972; Erhardt 1974; Ramirez et al.1994; Sanchez 1995; Veron 2000

Pfaff 1969; Antonius 1972; Erhardt 1974; Sánchez 1995; Ramirez et al. 1994 ;Veron 2000

Pfaff 1969; Ramirez et al. 1994; Sánchez 1995;Veron 2000 de Invertebrados Marinos. Ecorregiones: gua (Guajira); tay (Tayrona); mag (Magdalena); san (San Andrés); dar (Darién). 
collections. The Scleractinian coral list Cnidaria: Anthozoa reported for Colombia (2) includes specimens that are in the Museo de Historia Natural Marina de Colombia (MHNMC; Santa Marta - Colombia) as well as the samples from National Museum of Natural History (NMNH; Smithsonian Institution, Washington D.C. - U.S.A.). However, hermatypic corals collected in the colombian Caribbean were recently referenced to the Museo Javeriano de Historia Natural "Lorenzo Uribe S.J.”, (MUJ, Bogotá Colombia).

This note reports 129 biological records and provides a list of 29 species of hermatypic corals which were determined through synoptic keys (3) and with field visual guides, using diagnostic characters to identify coral recruits, $<4 \mathrm{~cm}$ diameter. This note remarks the information contained in the MUJ coral list: 1 . Seven exclusive species referenced for Colombia, which generates high taxonomic complementary (4) with respect to MHNMC and NMNH; 2. Twenty-three species of hermatypic coral recruits, $79 \%$ of the collection, makes this collection exclusive and unique in Colombia. Their skeleton and morphological diagnostic characters will make easier recruit identification (in situ) in future studies. Information contained in the MUJ coral list will complement other National marine collections that handle the same biological group, giving a precise dimension of Colombia biodiversity, and it will make easier future researches. It's important to clarify that Reyes list (2000) included Eusmilia fastigiata in the Caryophylliidae family, although it belongs to Meandrinidae (5). In the same way, the author overestimates Colombian biodiversity reporting Leptoseris and Helioseris as different genera, when Veron (2000) and other authors, i.e. Cairns 1999, only validate Leptoseris (5). In the Caribbean Veron (2000) reports the presence of Leptoseris cucullata, which in Colombia has been called Helioseris cucullata (6).

Key Words: Hermatypic Corals, MUJ Collection, Colombian Caribbean, Species List.

\section{Referencias}

1. Instituto de Investigaciones y Recursos Biológicos Alexander von Humboldt - IavH. Sistema de información en biodiversidad y sistemas de indicadores de seguimiento de política de biodiversidad. Bogotá, Colombia; 2002. En: www.humboldt.org.co/sib

2. Reyes J. Lista de los Corales Cnidaria: Anthozoa: Scleractinia de Colombia. Biota Colombiana 2000; 2: 164-176.

3. Veron J. Corals of the World. Australian Institute of Marine Science. Australia: Queensland, Old Pty Ltd. 2000;123-463,429490 p.

4. Colwell R, Coddington J. Estimating terrestrial biodiversity through extrapolation. 101-118p. In Hawksworth D.L. (ed): The Quantification and Estimation of organismal biodiversity. Special volume, Phil. Trans. R, Soc. London 1996. 101-118p.

5. Cairns S. Species richness of recent Scleractinia. Atoll Research Bulletin. 1999; 59:1-46.

6. Díaz J, Barrios L, Cendales M, Garzón J, Geister J, López V, G, Ospina G, Parra F, Pinzón J, Vargas F, Zapata F, Zea S. Areas coralinas de Colombia. INVEMAR, Ser. Publ. Espec. 2000; 5:176.

7. Geyer O. Vorläufige Liste der Scleractinien Korallen der Bahía de Concha bei Santa Marta, Kolumbien Mitt. Inst. Colombo-Alemán. Invest. Cient. 1999;3:17-24.

8. Pfaff. A. Los Scleractinia y Milleporina de las Islas del Rosario. Mitt. Inst. Colombo-Alemán. Invest. Cient.1969; 3:17-24.

9. Antonius A. Occurrence and distribution of Stony corals (Anthozoa and Hydrozoa) in the vicinity of Santa Marta, Colombia. Mitt. Inst. Colombo-Áleman. invest Cient. 6:89-103.

10. Werding B, Sánchez, H. The coral formations and their distributional pattern along a wave exposure gradient in the area of Santa Marta. Medio Ambiente. 1989;10(2):61-68.

11. Sánchez J. Benthic comunities and geomorphology of the Tesoro Island coral reef, Colombian Caribbean. An. Inst. Invest. Mar. Punta Betin. 199;24:55-77.

12. Ramírez A, Miranda D, Viña, G. Estructura arrecifal del archipiélago de San Bernardo (Mar Caribe, Colombia) estudio de línea base. Trianea. 1994; 5:189-220.

13. Zea S. Cover of Sponges and others sessile organisms in rocky and coral reef habitats of Santa Marta, Colombian Caribbean sea. Carib. J. Sci. 1993;29(1-2):75-88.

14. Erhardt H. Liste der Scleractinien Korallen der Bahía de Concha bei Santa Marta, Atlantikküste Kolumbien Senckenbergiana. Biológica. 1974; 55(4-6):399-408.

15. Prhal H, Erhardt H. Colombia Corales y Arrecifes Coralinos. FEN, Bogotá. 1985.252pp.

16. Blanco J, Díaz J, Ramírez G, Cortés, M. El banco de las animas: Una amplia formación arrecifal desarrollada sobre una antigua delta del río Magdalena. Boletín Ecotropica: Ecosistemas tropicales. 1994;27:10-18. 